\title{
Cognitive State Classification in a Spoken Tutorial Dialogue System
}

\author{
Tong Zhang ${ }^{1}$, tzhang1 @ifp.uiuc.edu \\ Mark Hasegawa-Johnson ${ }^{1}$, hasegawa@ifp.uiuc.edu \\ Stephen E. Levinson", sel@ifp.uiuc.edu
}

${ }^{1}$ Department of Electrical \& Computer Engineering

University of Illinois at Urbana-Champaign

405 N. Mathews Ave.

Urbana, IL 61801 USA 


\section{Abstract}

This paper addresses the manual and automatic labeling, from spontaneous speech, of a particular type of user affect that we call the cognitive state in a tutorial dialogue system with students of primary and early middle school ages. Our definition of the cognitive state is based on analysis of children's spontaneous speech, which is acquired during Wizard-of-Oz simulations of an intelligent math and physics tutor. The cognitive states of children are categorized into three classes: confidence, puzzlement, and hesitation. The manual labelling of cognitive states had an inter-transcriber agreement of kappa score 0.93. The automatic cognitive state labels are generated by classifying prosodic features, text features, and spectral features. Text features are generated from an automatic speech recognition (ASR) system; features include indicator functions of keyword classes and part-of-speech sequences. Spectral features are created based on acoustic likelihood scores of a cognitive state-dependent ASR system, in which phoneme models are adapted to utterances labelled for a particular cognitive state. The effectiveness of the proposed method has been tested on both manually and automatically transcribed speech, and the test yielded very high correctness: $96.6 \%$ for manually transcribed speech and $95.7 \%$ for automatically recognized speech. Our study shows that the proposed spectral features greatly outperformed the other types of features in the cognitive state classification experiments. Our study also shows that the spectral and prosodic features derived directly from speech signals were very robust to speech recognition errors, much more than the lexical and part-of-speech based features.

Keywords: intelligent tutoring system, user affect recognition, spoken language processing. 


\section{Introduction ${ }^{1}$}

\subsection{A Multimodal Spoken Tutorial Dialogue System}

An intelligent tutoring system (ITS) is a computer program that seeks to tutor users in an educational subject. The name intelligent tutor is used typically to label a system that is designed to behave as much as possible like a human tutor, rather than simply providing information and exercises like a textbook. The target application platform for the work described in this paper is an intelligent tutor in math and physics, using a Lego construction set, for children of elementary and early middle-school ages. The communication between the intelligent tutor and children users is through speech; meanwhile the intelligent tutor tracks the facial expression and activities of the users for a better estimate of the user state. The complete system has not been finished yet; the database used in this study was collected by Wizard-of-Oz simulations of the finished system, in which a human tutor (wizard) played the role of the intelligent tutor. The remainder of this section describes in detail the tutorial content, the Wizard-of-Oz experiments, and the characteristics of the tutoring dialogue scenario.

\subsubsection{Tutorial Content}

Our intelligent tutoring system is motivated by active learning, also called "learning by doing." Active learning originates from Constructionism (Kafai and Resnick, 1996), which proposes that children can learn knowledge not solely by straightforward instruction, but also by devising some types of external artifact. Figure 1 shows a child subject playing with the Lego set.

Researchers have found that some basic mathematical concepts can be acquired through manipulating objects rather than solely handling abstract symbols (Wilensky, 1991). Therefore, we use a novel method of primary education in mathematics-playing with concrete Lego gears. In each experiment, the user is given gears of different sizes. We expect students to learn some basic mathematical knowledge such as ratio and reciprocal by observing the spinning activities of the Lego gears. For example, one question is about the relationship between gear size and spinning speed: Line up a 24tooth gear and a 40-tooth gear. If the 24-tooth gear spins 5 times, then how many times

\footnotetext{
${ }^{1}$ Part of this work was presented at ICSLP 2004.
} 
must the 40-tooth gear spin for them to line up again? Why? Children can answer this question by spinning the gears and counting the cycles. To simplify counting of cycles, the teeth on each gear are painted with a pair of different colors: red and yellow, red and blue, or yellow and blue. The traditional approach of teaching children mathematics is through memorizing formulas and rules. The proposed intelligent tutor provides children with concrete objects (Legos) to help them develop a physical understanding of some abstract mathematical concepts.

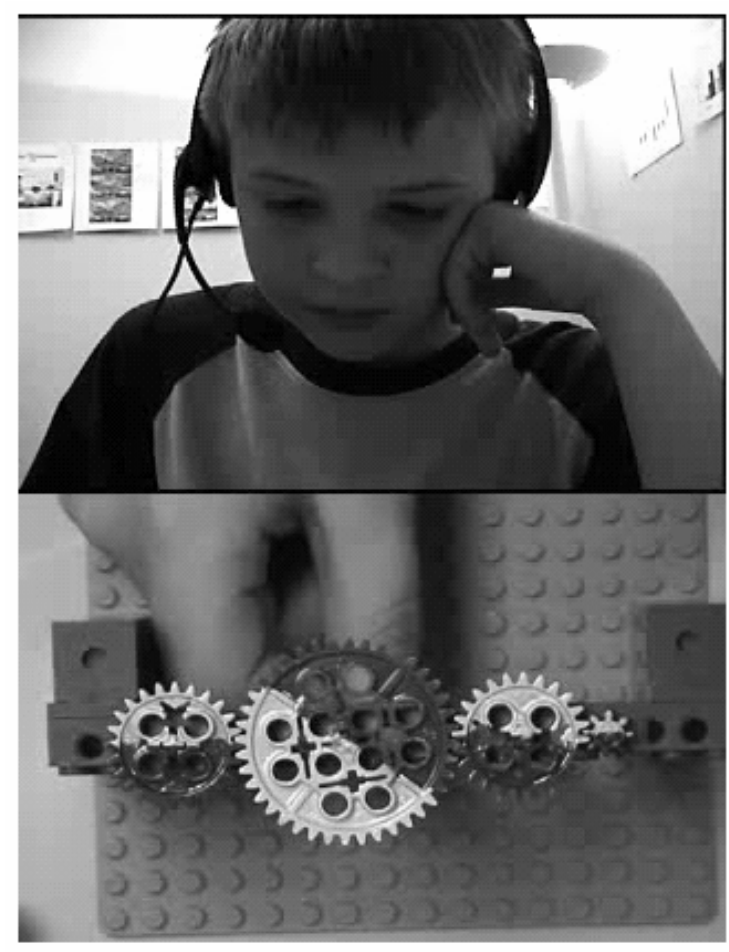

Figure 1: Graphic demonstration of the Lego playing scene (the lower picture is showing the Lego gears that the child in the upper picture is playing).

Physics is a discipline involving more direct interaction than mathematics with the physical world. The learning of some basic knowledge of physics can also be achieved by playing with Lego gears. For example, one physics question about interactive force is: Put one hand on the 40-tooth gear axle, and put the other hand on the 8-tooth gear axle. What happens if you hold one of them steady, and try to turn the other one? Why? Children usually think that the big gear is stronger before they do the experiment. 
However, it turns out that the small gear is "stronger," in the sense that it delivers greater force. Table 1 is an excerpt of the tutorial dialogue scenario.

(1) T: What are you exploring there?

(2) U: Seeing if the small gears move the big gear.

(3) T: What can you tell me about the directions they are spinning?

(4) U: They're moving in different directions mostly.

(5) T: What else do you notice?

(6) U: Big gears move in different ways and uhm ... with the first when you push one of the first gears, the other gear, the last gear moves, you know, and the gear after that moves then the gear after that one moves.

(7) T: What if we try just using three gears? What do you notice?

(8) U: They're moving in the same direction.

(9) T: What effect does the medium gear have?

(10) U: The medium gear is, it is stronger than the big one and it's smaller and weaker than the small one.

(11) T: Why is the smaller gear stronger?

(12) U: Their teeth are smaller. If you look at it very closely, then there's just a little bit more space.

Table 1: An excerpt of the tutorial dialogue scenario

\subsubsection{Multimodal Wizard-of-Oz Experiments}

System development data were acquired using a Wizard-of-Oz paradigm: children believed that they were communicating with a computer tutor instead of a human tutor, and therefore behaved as they would in a real computer-interaction environment. The system had multiple channels between student and tutor. On the one hand, the tutor used both visual objects and synthesized audio explanation to coach. On the other hand, the tutor listened to the student while tracking eye movement, body, a real-time video display of the gears, and facial expressions. 
In the experiments, the user and the tutor were sitting in separate rooms. The user orally communicated with a computerized talking head shown on the computer screen ahead of him using a head-set microphone. The lip movement of the talking head was coincident with speech synthesized from text that was typed by the tutor. Identical Lego gearsets were placed in front of both the tutor and the user. Video of the location and motion of the tutor's gearset was recorded by a digital camera and displayed to the user. Video of the user's gearset and face were both simultaneously recorded by a digital camera and displayed to the tutor, and the tutor was able to listen to the user. The user's eye movement and facial expressions were also captured by a digital camera and transmitted to the tutor's computer. An instrument consisting of a receiver installed on the user's back and a transmitter carried on the head of the user was used to track the user's body position. The tutor communicated with the user by typing questions, comments, and instructions; the tutor's utterances were displayed on the student's computer screen and played to the user in the form of synthesized speech. Figure 2 shows the screen display of the tutor's computer and the user's computer.
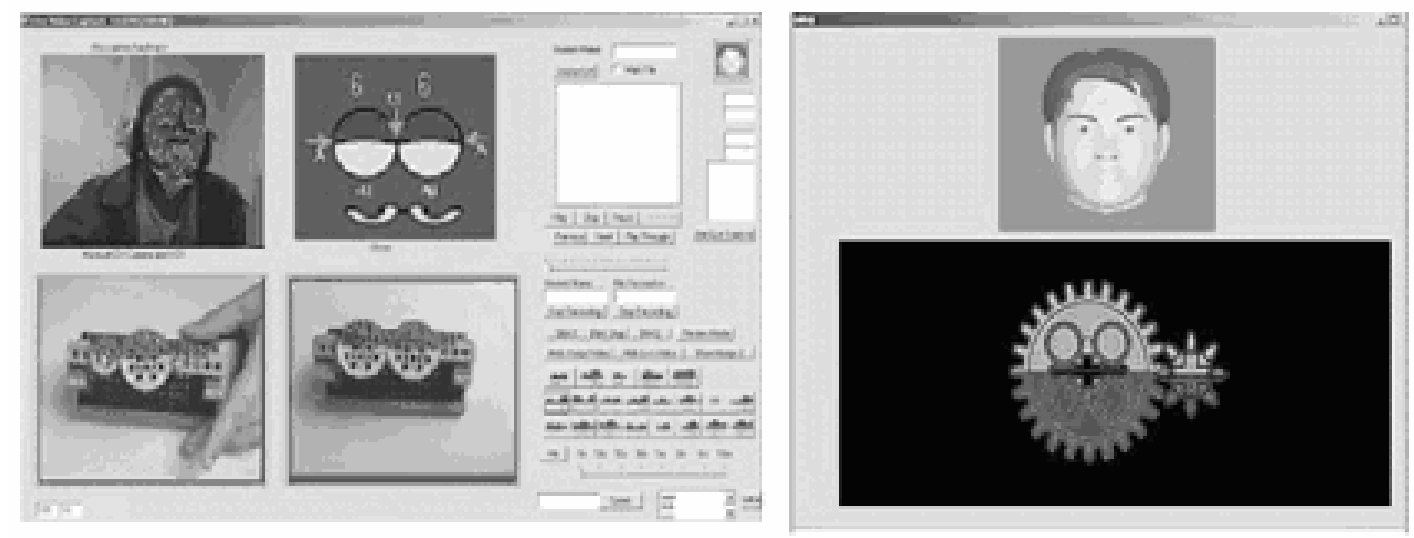

Figure 2: Screen displays on the tutor's computer (left) and the user's computer (right)

\subsubsection{Characteristics of the Dialogue Scenario}

The tutoring dialogue scenario has three characteristics:

1. Mixed-initiative interaction-Practice provides an efficient way for children to think and motivate new ideas by themselves. Therefore, in the experiments children 
take the initiative to discover and learn by themselves, while the tutor serves auxiliary functions, e.g., suggesting tasks and answering students' questions. The tutor usually does not override the wishes of students, but the tutor initiates communication when he finds that students need guidance, assistance or encouragement. Therefore, in the dialogue system the interaction is mixed initiative. For example, the tutor can ask elicitation questions to help students better understand the knowledge involved in the phenomenon they observe, or to suggest new actions for students to perform when a task is finished. Conversely, students can ask questions if they have trouble solving a puzzle, or if they fail to understand a tutor's utterance, or even if they dislike a puzzle that the tutor has set for them, and want to move on to something else.

2. Open-ended speech-Students are allowed to express their views unreservedly although the dialogues are in a limited domain. Educational psychologists find that it is hard to keep children interested in the experiments if their views and expression are constrained. So we encourage children to participate in the experiments and instigate their interests in scientific learning by asking them openended questions rather than single-choice questions. For example, the tutor prefers to ask "What are you noticing?" instead of "Which gear was turning faster?" Similar open-ended question examples are shown in the excerpt in Table 1, such as (1) What are you exploring there? (2) What if we try just using 3 gears? What do you notice? (3) What effect does the medium gear have? (4) Why is the smaller gear stronger?

3. Inexperienced users-Adult users of a typical telephone-based dialogue system (e.g., for purchase of air travel, train travel, or financial instruments) are usually able to learn, over a number of repeated interactions with the system, what actions are possible at each stage of the dialogue. By contrast, children users of our intelligent tutor are inexperienced with the content of the tutoring session. Each child participates in at most three sessions, and we do not ask children to relearn knowledge that they have acquired. Therefore, the children users are always inexperienced in the content of the lessons, even though they have gained some operational skills by using the system in the past. 


\subsection{User Affect in the Tutorial Dialogue System}

The internal state of a speaker, including such factors as strong emotion (e.g., angry, happy, fear), attention (e.g., relaxed, stressed, interested, bored), and attitude (e.g., formal, friendly, impatient), and information concerning the subject matter and the situation may be collectively referred to as affect (Gobl and Chasaide, 2003). Our preliminary analysis of the ITS corpus indicates that children do not exhibit strong emotions such as happy and angry. They also usually maintain great interest and attention during the experiments, so it is not usually possible (or necessary) to distinguish the attentional states such as interested vs. bored, and attitude such as friendly vs. hostile. Instead, their speech carries information closely related to the students' cognitive activities during the process of knowledge acquisition. Therefore, we call them cognitive states. For example, children's various answers to a question reflect the levels of their certainty; the questions they put forward reflect the fact that they have confusion.

We categorize the cognitive states of students into three classes: confidence, puzzlement, and hesitation. Generally, we classify the cognitive state to be confidence when the user answers questions or explains actions in relatively fluent speech (e.g., I was looking how many times the small gear goes around the medium and then the medium goes around the large and see if they end up the same), or issues commands (e.g., I lost count, let's try again), or explains the playing actions (e.g., I'm gonna move the two 24 gears close together), or expresses completion of a task (e.g., Alright, I'm done with that). We classify the cognitive state to be puzzlement when the user asks questions (e.g., How many times do both of the small tooth gears go around the medium size gear?), or states with certainty the lack of knowledge (e.g., I have no idea why this works). We classify the cognitive state to be hesitation when the user answers questions or explains actions in heavily dysfluent and relatively slow speech (e.g., Trains, oh, yeah, I mean on trains they have the...), or state uncertainty (e.g., I'm not sure).

Our proposed cognitive states classification is not only useful for friendly user interface design (as a type of affective computing), but also useful for detecting learning activities of students and thereafter selecting appropriate tutorial tactics. In this study, the learning activities of students are summarized by being categorized using a list of about 30 application-dependent classes, where the classes are defined in order to summarize:

(1) the application-dependent topic of the user's question; and (2) the implied state of the 
user's knowledge about the topic addressed. For example, we classify questions requesting instructions for playing with Legos into AskForPlayInstruction, classify those answers irrelevant to the experiment content into IrrelevantAnswer, and classify the utterances talking about the spinning speed into SpinSpeed. Many learning activities are typically associated with particular cognitive states. For example, hesitation is more likely to accompany IncompleteAnswerOnSpin (e.g., I saw the ... yellow part ...) or WrongAnswerOnSpin (e.g., It ... I think it goes around ... one and a half times) than CorrectAnswerOnSpin (e.g., The large gear has five times as many teeth as the small ones); puzzlement is a strong indicator of a question such as AskForPlayInstruction.

In this study, we intend to examine: (1) the efficiency of manual and automatic labeling of the cognitive states, i.e., confidence, puzzlement, and hesitation, that we define in a tutorial dialogue system; (2) speech features such as spectrum, syllabic rate and pitch, and language features such as word choice and word combination, that may reflect children users' cognitive states; and (3) the robustness of speech and language features to speech recognition errors.

\section{Related Work}

\subsection{Intelligent Tutors}

Automatic intelligent tutors have been a topic of research and development for many years, and have been used for instructional purposes in many academic disciplines. For example, an intelligent tutor has been used for teaching context-free grammar through instruction and remediation (Reyes et al., 2000), an intelligent tutor has been used for teaching microscopic diagnosis by viewing virtual pathology slides (Crowley et al., 2003), and an intelligent tutor has been used for apprenticing Smalltalk programmers (Alpert et al., 1999). Some intelligent tutors have been shown to accelerate the learning efficiency of students. For example, an Air Force electronics troubleshooting tutor allowed students to gain proficiency in two weeks equivalent to the proficiency of 48month trainees without ITS (Lesgold et al., 1990). A LISP tutor improved the speed and quality of students' programming in comparison with traditional classroom training (Corbett and Anderson, 1992). Aimed at helping college students master basic computer techniques, the AutoTutor system (Graesser, 2001) showed that students can improve 
their learning by 0.5 standard deviation units compared with learning by reading alone. In addition, it has even been proposed that ITS has advantages over classroom instruction, in particular, the ITS educational environment is not competitive, and thus benefits the students with special needs. For example Discover, an intelligent tutor, taught students with learning difficulties to solve mathematical word problems with less failure or frustration (Steele and Steele, 1999).

Most tutorial dialogue systems rely on text input from students, but a few tutoring systems have experimented with the use of speech in tutorial dialogues. CLT (Colorado Literacy Tutor) applies spoken dialogue system techniques in CU Communicator (Ward and Pellom, 1999; Pellom et al., 2000) to teach vocabulary to children with hearing problems and autism spectrum disorders (Cole et al., 2003). The project LISTEN intends to develop an automatic reading tutor to anticipate, detect, and remediate the difficulties of students in reading (Mostow et al., 2002; Beck et al., 2003). ITSPOKE is a spoken tutorial system that engages students in qualitative physics learning by providing feedback and correcting misconceptions, and eliciting more complete explanations (Litman and Silliman, 2004). SCoT is a spoken conversational tutor that utilizes speech recognition and natural language understanding techniques to identify and address students' misconceptions for a shipboard damage control simulator (Clark et al., 2001; Pon-Barry et al., 2004; Schultz et al., 2003). A recent study comparing speech-based versus text-based tutorial found that speech has advantages over text in the learning gains and time required to accomplish a task when the tutor was human, but little difference between the two kinds of tutorial strategies when the tutor was a computer (Litman et al., 2004). This experimental result suggests that a perfect spoken tutorial would be more efficient than a typed tutorial, but that state-of-the-art speech techniques are not mature enough to guarantee that the spoken tutorial outperforms the typed tutorial.

\subsection{Affective Computing in Spoken Dialogues}

Speech conveys a variety of affective information that can be used to improve the naturalness and friendliness of user interface for human computer communication. Behavioral results show that an agent aware of user affect is significantly more effective than a neutral agent in helping relieve frustration levels. For example, in an automatic call center it is very useful to detect user affect such as frustration, irritation or 
impatience, so that a call can be redirected to a human attendant in time (Lee and Narayanan, 2005; Petrushin, 1999; Batliner et al., 2003). Many breakdowns in a telephone-based information system could be avoided if the machine was able to recognize the emotional state of the user, such as annoyance and frustration, and responded to it more sensitively (Martinovsky and Traum, 2003; Ang et al., 2003). Fernandez and Picard (2003) classify driver's speech into four levels of stress in terms of driving speed and frequency of proposing arithmetic questions. A tutorial dialogue system classifies user emotion into positive, neutral, and negative (Forbes-Riley and Litman, 2004) for better user modeling.

Affective activity causes physiological variations in the vocal mechanism, which is used to generate sound and causes further speech variation. The audio speech waveform carries various kinds of information that reveal user affect: long-term prosody, short-term spectrum, lexicon, syntax, and implications that carry meaning because of their relationship to dialogue context. (1) Prosody revealing phonetic variations is most commonly used for user affect recognition. Pitch is the most relevant acoustic parameter for the detection of emotion (Mozziconacci and Hermes, 1998; Juang and Furui, 2000; Petrushin, 2000; Kang et al., 2000). For example, aroused emotions (such as fright and elation) are correlated with relatively high pitch, while relaxed emotions (such as boredom and sadness) are correlated with relatively low pitch. The other prosodic features are energy, duration, and speaking rate. (2) Certain word choices and word combinations are more likely to express certain affect. Lexical information has been encoded, for the purpose of affect recognition, in the form of affect-dependent unigram and bigram language model statistics (Batliner et al, 2000; Polzin and Waibel, 2000). Lee and Narayanan (2005) used word-emotion mutual information to automatically detect a set of emotionally salient words. (3) Discourse provides a knowledge source to help reveal emotion (Batliner et al., 2003; Lee and Narayanan, 2005). For example, the repetition of the same dialogue act is likely to indicate trouble in communication.

\section{Corpus Description}

To date 29 experiments with 17 subjects have been carried out and transcribed, and we have collected $11.7 \mathrm{hrs}$ of audio-visual data. Subjects ranged in age from 9 to 12. Some 
student subjects spent the entire tutoring session playing silently with the Legos, and declined to respond even when the tutor tried to engage them in conversation. However, some students were relatively loquacious, and provided us with a relatively rich speech database for our study. We manually extracted some utterances from users' speech that could reflect the users' cognitive activities. The database did not include back channels and "neutral" utterances such as $o k$, thanks, that's nice, etc. In addition, some data had to be discarded because of Lego block noise, heavy breathing, etc. To date we have collected 714 student utterances, containing approximately 50 minutes of relatively clean speech. On average each utterance had $4.2 s$ speech and 8.1 words.

Three annotators worked on the Wizard-of-Oz audio data independently of each other. The annotation was performed based on speech content and dialogue context. The consistency among the annotators was annotated by the Kappa statistic, $K=\left(P_{O^{-}} P_{C}\right) /(1$ $P_{C}$ ), where $P_{\mathrm{O}}$ is the percent of times when the annotators agree, and $P_{\mathrm{C}}$ is the percent of times when the annotators are expected to agree by chance (Flammia, 1998). Kappa is 1.0 when the annotators agree on all transcriptions, and is 0.0 when the rate of agreement equals the average rate that would be achieved by chance. The kappa statistics on our corpus annotation yielded a score of 0.93 , indicating a very good agreement. Compared with the inter-speaker agreement on emotion labeling achieved by Forbes-Riley and Litman (2004), Ang et al., (2002), etc., our annotator agreement was very high. This might because according to the annotation criteria, the distinction among the three cognitive states was more explicit than the distinction among the delicate and subtle emotional states. Therefore, the annotators were easy to make agreement on labeling. We used majority voting to resolve the annotation differences among the three annotators: for each utterance, if two or more annotators had the same label, then we assigned that label to the utterance, otherwise (i.e., each annotator had a different label) the utterance was removed from the corpus. Table 2 shows the distribution of utterances in our corpus with respect to the cognitive state classes.

Table 2: Cognitive state statistics

\begin{tabular}{lccc}
\hline & Confidence & Puzzlement & Hesitation \\
\hline \# of Utterances & 441 & 216 & 57 \\
\hline$\%$ of All Utterances & 61.8 & 30.2 & 8.0 \\
\hline
\end{tabular}




\section{Proposed approach}

\subsection{Overview of the Cognitive State Classifier}

Our cognitive state classifier is based on the analysis of a large number of speech-related and language-related information sources, including long-term prosody, short-term spectrum, lexical features (word choice), and syntactic features (part-of-speech sequence). First, prosody conveys paralinguistic information about cognitive activities. For example, hesitant speech tends to have lower signal energy and longer phonetic word boundaries, while puzzled speech is often associated with raised pitch in the utterancefinal syllable, especially but not exclusively if the utterance is a yes/no question. Second, some utterances are inherently ambiguous for cognitive state detection by means of prosody alone. For example, wh-questions and no-opinion statements (e.g., 'I don't know' denotes puzzlement, and 'I'm not sure' denotes hesitation) may be uttered with prosody indistinguishable from the prosody of a confident utterance. Spotting some key words/phrases embedded in the fluent speech helps to identify these special cases. Third, the cognitive state affects the articulation of phonemes, thus the spectra of speech signals may indicate the cognitive states. Fourth, we notice that speakers in confident mode and puzzled mode usually use different linguistic structures, while hesitant utterances are often ungrammatical and incomplete. For example, a wh-question (usually indicates puzzlement) has different syntactic structure than a statement (often indicates confidence).

The overall structure of the cognitive state classifier is depicted in Figure 3. An automatic speech recognition (ASR) system first transcribes the utterance; word error rate of the ASR is typically high. Features for cognitive state classification are extracted as follows. First, lexical features are extracted from the recognized word string output of the ASR system. Second, the recognized word string is automatically tagged with part-ofspeech, and the part-of-speech sequence is extracted as syntactic features. Third, the speech waveform and the transcribed/recognized word duration are used by the prosody analyzer: some prosodic features depend only on the speech waveform, but some also depend on word boundary alignment duration. Fourth, spectral evidence is obtained by passing the speech signal through three cognitive state-dependent ASR systems. The cognitive state-dependent ASR system is the same as the routine ASR system except that the former uses phone models that are adapted to data categorized into cognitive classes. 
The corresponding acoustic log likelihood scores based on different cognitive state assumptions are used as the spectral evidence. Finally all the analytical results go into a classification decision tree, which determines the cognitive state that maximizes the likelihood of the given lexical, prosodic, spectral, and syntactic evidences.

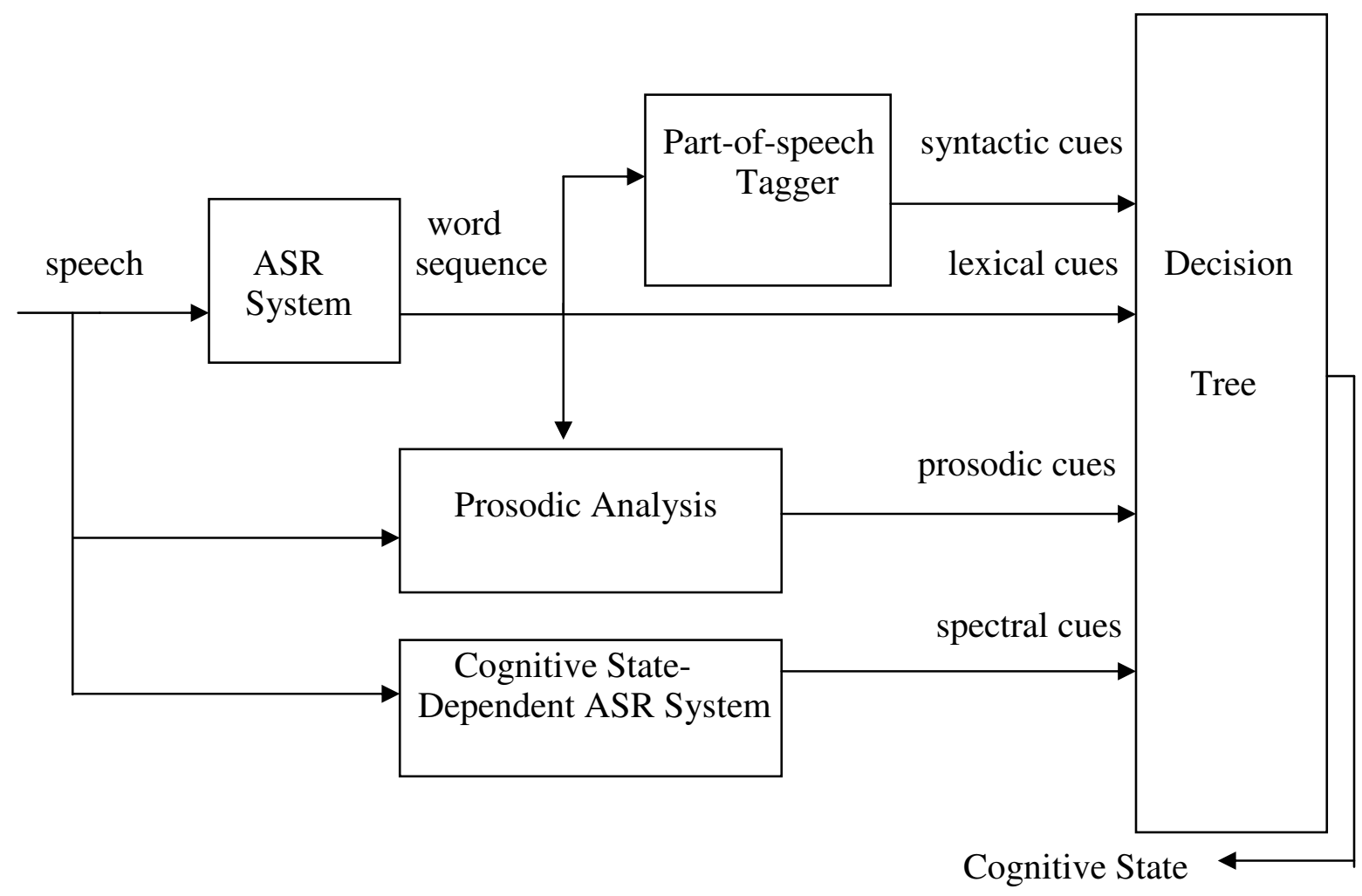

Figure 3: Architecture of the cognitive state classification system

\subsection{Children Speech Recognition}

\subsubsection{Acoustic Analysis of Children's Speech}

Children's speech has very different acoustic characteristics than adults' speech. The vocal tracts of children are short and still growing. The shorter vocal tract length makes formant frequencies of children higher than those of adults. Speech of children under 13 years old exhibits both high inter-speaker and high intra- speaker variabilities, leading to extremely high speech-to-text word error rate (Narayanan and Potamianos, 2002). The inter-speaker variability includes age-dependent variability of formant frequencies, and 
unusually high variability among speakers of the same age caused by factors including the different growth rates of different children. The intra-speaker variability is caused by both developmental factors (children are less likely than adults to repeat an utterance with exactly the same articulation, so the spectral envelope shape both within a token and across two repetitions has larger variations) and signal processing factors (the high $F 0$ of a child means that formant peaks are not well represented in the spectrum, and thus there is ambiguity in the mapping from cepstra to phones). These acoustic characteristics of children's speech result in degradation of ASR performance on children's speech. It has been reported that the in-vocabulary word error rate of children's speech is almost twice that of adults' speech (Zue et al., 2000).

We apply frequency warping (Lee and Rose, 1998; Zhan and Westphal, 1997) to normalize the vocal tract length of children, as shown in Figure 4. The frequency $f$ is warped by means of a bilinear transform, which maps an input to an equal length of output in the frequency domain:

$$
\varphi_{\beta_{f}}(f)=f+\frac{2 f_{N}}{\pi} \tan ^{-1}\left(\frac{\left(1-\beta_{f}\right) \sin \left(\frac{f}{f_{N}} \pi\right)}{1-\left(1-\beta_{f}\right) \cos \left(\frac{f}{f_{N}} \pi\right)}\right),
$$

where $f_{N}$ is the Nyquist frequency, and the warping factor $\beta_{f}$ is not constant but dependent on frequency. To compensate for the inter-speaker and intra-speaker variabilities, different warping factors are used on groups of children with the same age and gender: with reference to the published data (Lee et al., 1999), for each group, the warping factors at formants $F_{1}, F_{2}$ and $F_{3}$ are computed as the ratio of average formant values of that group to those of adult males; the warping factors for frequencies other than the three formants are approximated by piecewise linear interpolation.

\subsubsection{Language Modeling}

The construction of a bigram language model usually requires millions of words; the data available to us from the ITS Wizard-of-Oz simulations is far less than that amount. To make up for data sparsity, we derive linguistic information on the part-of-speech level from the Switchboard transcription (Godfrey et al., 1991) whereby to establish the word- 
level language model for our system. Our approach first promotes the words in our system vocabulary onto their part-of-speech tags. A word can have several part-of-speech tags. We consider all the possible part-of-speech tags for a given word, and the probability of attaching a part-of-speech tag to that word is derived from the part-ofspeech tagged Switchboard transcription. Next we compute the part-of-speech bigram probabilities based on the Switchboard transcription using the backoff smoothing technique (Katz, 1987). The part-of-speech tags of the transcription data are determined by an automatic tagger (Munoz et al., 1999). Then we apply the part-of-speech linguistic information to the system to derive a word-level bigram. Specifically, the word bigram model is given by:

$$
p\left(w_{2} \mid w_{1}\right)=\sum_{P O S_{2}, P O S_{1}}\left\{p\left(P O S_{1} \mid w_{1}\right) p\left(\left(P O S_{2} \mid P O S_{1}\right) p\left(w_{2} \mid P O S_{2}\right)\right\}\right.
$$

where $w_{1}$ and $w_{2}$ are two words in the vocabulary of our dialogue system, $\mathrm{POS}_{1}$ and $\mathrm{POS}_{2}$ are all possible part-of-speech tags of words $w_{1}$ and $w_{2}$, respectively, $p\left(\mathrm{POS}_{1} \mid w_{1}\right)$ is the empirical frequency with which $\mathrm{POS}_{1}$ is attached to $w_{1}$ in the Switchboard transcription, $p\left(\mathrm{POS}_{2} \mid \mathrm{POS}_{1}\right)$ is the part-of-speech bigram probability in the Switchboard transcription, and $p\left(w_{2} \mid \mathrm{POS}_{2}\right)$ is the empirical frequency of $w_{2}$ given $\mathrm{POS}_{2}$ in the ITS Wizard-of-Oz speech data.

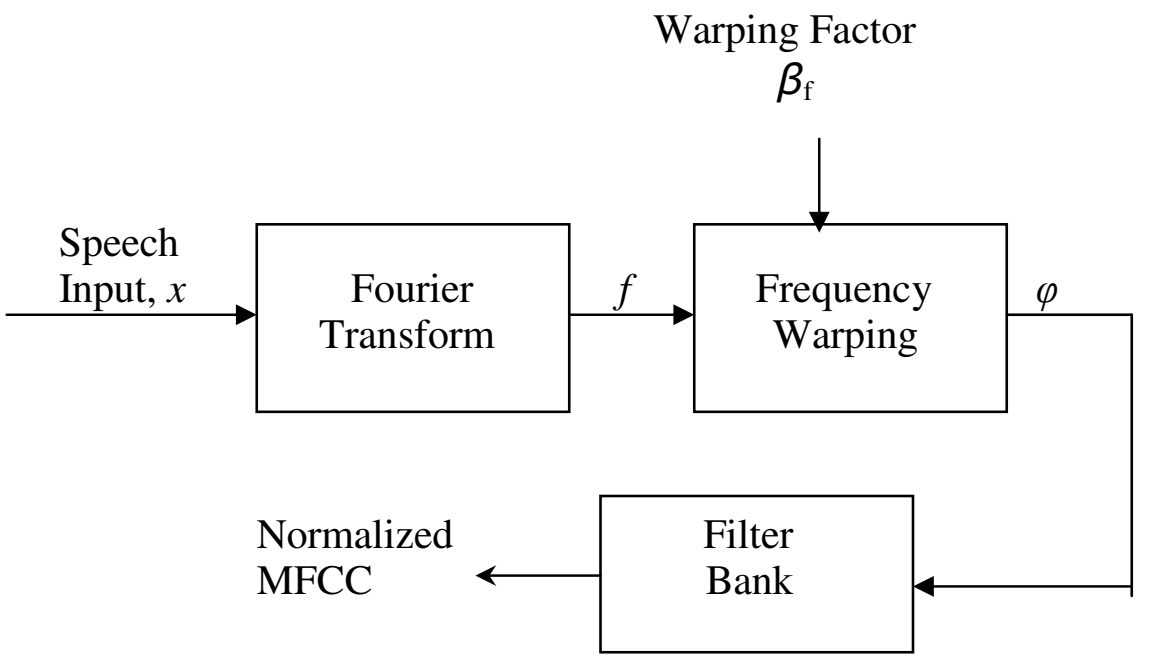

Figure 4: Vocal tract length normalization by frequency warping 


\subsection{Lexical Analysis}

Some keywords are closely associated with particular cognitive states. For example, filled pauses (e.g., 'uhm' and 'ahh') are likely to denote hesitation; wh-words such as 'what' and 'when' are likely to denote puzzlement. Data sparsity makes it impractical for us to automatically generate a keyword list. Therefore, we manually generate a list of approximately $50 \mathrm{key}$ words/phrases that are affectively salient. In order to further avoid problems of data sparsity, the 50 key words/phrases are grouped into 9 keyword classes. The spotted key word(s)/phrase(s) from a recognized word string is (are) assigned to corresponding keyword classes, resulting in a 9-dimensional vector of binary indicator variables denoting the presence or absence of each keyword class. Table 3 lists all of the keyword classes and their keyword components.

Table 3: Keyword classes and component keywords

\begin{tabular}{|cl|}
\hline Keyword Class & \multicolumn{1}{c|}{ Keywords } \\
\hline Affirm & yes, yeah, yep, no \\
\hline Digit & one, two, three, etc \\
\hline Known & I know, I believe \\
\hline Uhm & ahh, ahm, ahmm, uhm \\
Reason & because, so \\
\hline Unknown & don't know, don't understand \\
Auxiliary & can I, can you, could you, do I, do you, should I, \\
& should we, would it, would you, would it \\
\hline Uncertain & not sure, not exactly sure \\
\hline Wh-word & how, what, when, where, which, why \\
\hline
\end{tabular}

\subsection{Prosodic Analysis}

As summarized in Table 4, the prosodic feature set includes:

Pitch-related features: Pitch and probability of voicing are estimated using the FORMANT program in Entropic XWAVES. Pitch measurements are ignored in all frames with a low probability of voicing. Valid pitch measurements are further standardized by the pitch range of the utterance:

$$
\text { pitch }_{f}=\frac{\text { pitch }_{f}-\min _{f}\left(\text { pitch }_{f}\right)}{\max _{f}\left(\text { pitch }_{f}\right)-\min _{f}\left(\text { pitch }_{f}\right)}
$$


where $\operatorname{pitch}_{f}$ is the pitch of the $f^{\text {th }}$ frame, $\min _{f}\left(\right.$ pitch $\left.h_{f}\right)$ is the minimum non-zero pitch value of the entire utterance, and $\max _{f}\left(\right.$ pitch $\left._{f}\right)$ is the maximum pitch value of the entire utterance. We compare pitch in the end region (the final $100 \mathrm{~ms}$ ) and the penultimate region (the previous $100 \mathrm{~ms}$ ), as well as looking at the least-squares regression lines covering the two regions.

Energy-related features: Typically, an utterance falls to lower energy when close to completion. When an utterance ends in a sentence fragment, this fall has not yet occurred and thus energy remains high (Jurafsky et al., 1997). Therefore, the comparison of energy in the final and penultimate regions can be indicative of hesitation. Energy is measured on a log scale and then peak-normalized. The ratio of average energy in the final and penultimate regions of an utterance, and the first and second order time derivatives of energy in the final region are used as features.

Pause-related features: As a cue to hesitation, pause refers to a time period of nonspeech lasting more than $600 \mathrm{~ms}$. Non-speech periods of less than $600 \mathrm{~ms}$ are not called pause, here, because they are more likely to be minor disfluencies and intonational-phrase-final junctures, while longer pauses tend to mark perceptually prominent disfluencies and hesitation. To detect a pause, energy is first passed through an anti-symmetric edge-detection filter, and then is compared with a timevarying threshold (Li et al., 2002). Pauses of longer than 600ms are extracted, their total duration is computed, and then normalized by the utterance duration.

Syllabic rate-related features: Usually people speak more slowly when they are hesitant and puzzled, as opposed to when they are confident. On average, the speaking rate of children is slower than that of adults. In addition, the speaking rate usually varies from person to person. Therefore, the syllabic rate of an utterance is divided by the speaker's normal syllabic rate, which is averaged from the speaker's utterances. In this study, syllabic rate is the average value of three estimators: (1) peak counting of the wide-band energy envelope followed by utterance duration normalization; (2) pointwise correlation between pairs of subband energy envelopes, followed by peak counting and utterance duration normalization (Morgan and Fossler-Lussier, 1998); and (3) peak frequency of the energy modulation spectrum (Kitazawa et al., 1997). 
Word-duration and utterance-duration features: Normal prosodic effects (prosodic phrase position and phrasal prominence) can lengthen a word by up to roughly $100 \%$; duration increases of more than about $100 \%$ are likely to indicate hesitation. The duration feature in an utterance is characterized by a vector of four measurements: average and maximum of the normalized word durations (the duration of a word is derived based on forced alignment and then normalized by the number of syllables in the word), syllable-normalized utterance duration, and wordnormalized utterance duration.

Table 4: List of the prosodic features

\begin{tabular}{|c|c|}
\hline Feature & Description \\
\hline F0_ratio & $\begin{array}{l}\text { Ratio of mean } F 0 \text { over the end region (the final } 100 \mathrm{~ms} \text { ) } \\
\text { and the penultimate region (the previous } 100 \mathrm{~ms} \text { ). }\end{array}$ \\
\hline F0_reg_pen & $\begin{array}{l}\text { Least-squares all-points regression over the penultimate } \\
\text { region. }\end{array}$ \\
\hline F0_reg_end & Least-square all-points regression over the end region. \\
\hline F0_norm & $\begin{array}{l}\text { The number of nonzero } F 0 \text { frames normalized by the } \\
\text { utterance duration. }\end{array}$ \\
\hline loge_ratio & $\begin{array}{l}\text { Ratio of logarithmic energy over the end region and the } \\
\text { penultimate region. }\end{array}$ \\
\hline derive_logE & $\begin{array}{l}\text { Mean of peak-normalized logarithmic energy derivative } \\
\text { over the end region. }\end{array}$ \\
\hline acce_logE & $\begin{array}{l}\text { Mean of peak-normalized logarithmic energy acceleration } \\
\text { over the end region. }\end{array}$ \\
\hline norm_pause & $\begin{array}{l}\text { Total pause durations normalized by the utterance } \\
\text { duration. }\end{array}$ \\
\hline Syllarate & $\begin{array}{l}\text { Syllabic rate normalized by the speaker's normal speaking } \\
\text { tempo. }\end{array}$ \\
\hline mean_norm_word_dur & $\begin{array}{l}\text { Mean of word duration which is normalized by the } \\
\text { number of syllables the word has. }\end{array}$ \\
\hline $\begin{array}{l}\text { max_norm_- } \\
\text { word_dur }\end{array}$ & $\begin{array}{l}\text { Maximum of word duration which is normalized by the } \\
\text { number of syllables in that word. }\end{array}$ \\
\hline utt_dūr_by_syllable & $\begin{array}{l}\text { Utterance duration normalized by the number of syllables } \\
\text { in that utterance. }\end{array}$ \\
\hline utt_dur_by_word & $\begin{array}{l}\text { Utterance duration normalized by the number of words in } \\
\text { that utterance. }\end{array}$ \\
\hline max_abs_word_dur & Maximum of absolute word duration. \\
\hline
\end{tabular}

\subsection{Spectral Analysis}

Spectrum captures vocal-tract movements. Articulatory movements may be rapid or slow, extreme or reduced, depending on the level of arousal of the speaker; changes in the rate 
and extremity of articulation cause corresponding changes in the spectral correlates of phonemes. This hypothesis is verified by a study of Lieberman and Michaels (1962), which showed that human listeners can recognize emotional speech segments with an accuracy of $85 \%$, but the accuracy decreased dramatically to just $47 \%$ when the spectral information was filtered out and only pitch and intensity information was preserved. The spectral feature is the least frequently employed feature in published studies of affect recognition. In previous studies the only case of incorporating spectral features into emotion recognition is the study of Polzin and Waibel (2000), who used an HMM-based speech recognition system to classify emotion. Polzin and Waibel modeled spectral information using a triphone Gaussian mixture model, in which each phone has 3 states, and each state observes 32 mel-frequency cepstral coefficients (MFCCs). The study did not show a significant contribution of spectral information to emotion recognition.

In this study, we propose a new approach for incorporating spectral information into user affect recognition. The short-term spectral envelope and its first and second temporal derivatives capture the dynamics of phoneme articulation. To manifest the influence of cognitive states on phoneme articulation, we adapt phoneme models using utterances labeled for the three cognitive state categories. For example, the confidencedependent phoneme models are adapted using the subset of training utterances from the ITS corpus that have been labeled as confidence. For a given utterance, ASR based on each cognitive state-dependent phoneme model generates a word string associated with an acoustic likelihood score. Each acoustic likelihood score spans a wide range. We compare the acoustic likelihood scores based on different cognitive state assumptions, and use their differences as the spectral cues. The spectral features are listed in Table 5.

Table 5: List of the spectral features

\begin{tabular}{cl}
\hline Feature & \multicolumn{1}{c}{ Description } \\
\hline Spect $_{\mathrm{c}}$-Spect & \multicolumn{1}{c}{$\begin{array}{l}\text { ASR log likelihood using the confidence-adapted phone } \\
\text { models minus ASR log likelihood using the puzzlement- } \\
\text { adapted phone models }\end{array}$} \\
\hline Spect $_{\mathrm{c}}$-Spect $\mathrm{h}_{\mathrm{h}}$ & $\begin{array}{l}\text { ASR log likelihood using the confidence-adapted phone } \\
\text { models minus ASR log likelihood using the hesitation- } \\
\text { adapted phone models }\end{array}$ \\
\hline Spect $_{\mathrm{p}}-$ Spect $_{\mathrm{h}}$ & $\begin{array}{l}\text { ASR log likelihood using the puzzlement-adapted phone } \\
\text { models minus ASR log likelihood using the hesitation- } \\
\text { adapted phone models }\end{array}$ \\
\hline
\end{tabular}




\subsection{Syntactic Analysis}

The syntactic composition of sentences is useful to reveal cognitive state. Puzzlement is often expressed in questions while confidence is often expressed in statements. Questions and statements can be distinguished, with reasonable accuracy, based on partof-speech of the first three words of the utterance. Sentences expressing hesitation are often ungrammatical or incomplete. The final word, or the exit of a sentence, is especially useful in detecting the completeness or incompleteness of a sentence. For example, an exit word with a conjunction part-of-speech usually indicates that the sentence is incomplete. The context of the exit word usage is also important. For example,

This gear is a much bigger

This gear is much bigger

The first sentence is incomplete while the second sentence can be considered complete, although they have the same exit word.

Spoken language usually contains ungrammatical junctures and dysfluencies, and therefore a complete syntactic parse of a spoken utterance may be inefficient or impossible. Instead, we use the part-of-speech of the first three words and the last three words of a sentence as the syntactic cues for cognitive state classification. These six words, as shown in our corpus analysis, rarely contain dysfluencies; information about these six words is rarely sufficient to reconstruct a complete parse of the sentence, but the correct part-of-speech of these six words is often sufficient to correctly classify cognitive state of the user. Part-of-speech tagging is performed by an automatic tagger (Munoz et al., 1999).

\section{System Evaluation}

\subsection{Children's Speech Recognition}

We designed a triphone-based ASR system with the help of the HTK toolkit (Young et al., 2000). Sampled at $11 \mathrm{KHz}$, the speech input was pre-emphasized and grouped into frames of 330 samples with a window shift of 110 samples. The speech signal was characterized by 13 MFCCs normalized by cepstral mean subtraction and log-scaled energy normalized by the peak. MFCCs, energy, their deltas and delta-deltas together 
formed a vector of 42 features. In the system, each word was represented by the concatenation of the models of its component phones. Each phone model was a left-toright 3-state HMM with an output distribution of 16 Gaussian mixtures per state. The speaker-independent universal background phoneme model was trained from the TIMIT database. Recognition was accomplished by a frame synchronous token-passing search algorithm to determine the sequence of words that maximized the likelihood of a given utterance.

Because of the limited size of the database, the 17 children speakers were divided into 6 groups in terms of age and gender (see Table 6). As what we describe in Section 4.2.1, children's speech has high inter-speaker variability. The age-dependent formants introduce variability in spectral features across the age groups. So if a phoneme model for ASR is based on a certain age group and tested against another age group, the mismatch between training and test data will result in degradation. According to the study of Lee et al. (1999), pitch and formant frequencies of male and female speakers become distinct at age 11. Therefore, gender is not a factor causing the degradation in speech recognition for children of 9-10 years old.

The universal background phoneme model trained from TIMIT was adapted to the groups of children, using about half of the total speech of each group, based on maximum likelihood linear regression adaptation followed by maximum a posteriori adaptation. The recognition performance is listed in Table 6. The low recognition accuracy on the one hand was caused by the acoustic characteristics of children's speech as we have described, and on the other hand was caused by the linguistic correlates of children's speech. Children's speech has higher degree of spontaneity than adults' speech. Children's speech is prone to dysfluency containing mispronunciation, false starts, breath noise, and filled pauses, more obvious with children younger than 11 years old (Potamianos and Narayanan, 2003). Since the children users in our system were not familiar with the experiment contents, their utterances were even more incoherent and dysfluent than those of typical dialogue system users, e.g., Ahmm when you... after it goes around once, the other one goes around the same, the same, I mean it goes around... you know you only have to spin it around once, and that makes sense basically because they are the same size. The language characteristics of children's speech in our system caused the language model perplexity to be as high as 593.42. 
Table 6: Speech recognition rate of children, by age group (vocabulary size $=515$ ). Test is against the total minutes of speech, including the adaptation data.

\begin{tabular}{|c|c|c|c|}
\hline Age & Gender & Total Minutes (Adaptation Minutes) & Recognition Accuracy, \% \\
\hline 9 & M \& F & 3.68 mins (1.8 mins) & 34.45 \\
\hline 10 & M \& F & 10.14 mins (5 mins) & 40.27 \\
\hline \multirow{2}{*}{11} & M & 11.02 mins (5 mins) & 61.58 \\
\cline { 2 - 4 } & F & 5.99 mins (3 mins) & 50.13 \\
\hline \multirow{2}{*}{12} & M & 13.61 mins (6mins) & 60.43 \\
\cline { 2 - 4 } & F & 2.99 mins (1.5 mins) & 54.20 \\
\hline
\end{tabular}

\subsection{Cognitive State Classification Performance}

The information sources were integrated probabilistically via a classification decision tree. We chose to use a decision tree classifier because of its efficiency in handling samples with high dimensionality, mixed data types, and nonstandard data structure. For the decision tree program, we used See5, which is a data mining tool for discovering patterns or relationships in data, assembling them into classifiers that are expressed as decision trees or sets of if-then rules, and using them to make valid predictions (Rulequest Research, 2004). Each variable in the feature vector $\mathbf{x}=\left(x_{1}, x_{2} \ldots x_{\mathrm{n}}\right)$ was in continuous, discrete, or character type. There were a total of 33 features, as listed in Table 7. The See5 program was invoked with the options rulesets (meaning that treebased classifiers were backed off into collections of if-then rules), boost (See5 generates several classifiers, whose classifications are weighted and summed to determine the predicted class), ignore costs file (so that the training algorithm gave equal weight to false positive and false negative errors), and global pruning (a large tree is first grown to fit the data closely and is then pruned by removing parts that are predicted to have a relatively high error rate).

We randomly chose 400 (56\%) samples for training and the remaining 314 (44\%) samples for evaluation; this process was repeated 10 times, and the results averaged (10fold cross-validation). Our study showed that for syntactic analysis, counting only the last word (exit word) yielded slightly better results than processing the last three words in an utterance, thus we recorded the result in the former case. 
Table 7: List of all features used for cognitive state classification

\begin{tabular}{|c|c|c|}
\hline Feature & Size & Description \\
\hline Prosody & 14 & $\begin{array}{l}\text { Continuous: } 4 \text { pitch-related; } 3 \text { energy-related; pause; syllabic } \\
\text { rate; } 5 \text { duration-related. }\end{array}$ \\
\hline Lexicon & 9 & Binary: absence vs. presence of each keyword class. \\
\hline Part-of-speech & 6 & $\begin{array}{l}\text { Character: either a character such as } V B P \text {, or a blank in the } \\
\text { case that the utterance is shorter than } 6 \text { words. }\end{array}$ \\
\hline Spectrum & 3 & Continuous \\
\hline Target & 1 & Discrete: $1=$ confidence, $2=$ puzzlement, $3=$ hesitation. \\
\hline
\end{tabular}

\subsubsection{Transcribed Speech}

We first tested cognitive state classification using manual transcriptions of the Wizard-of$\mathrm{Oz}$ corpus. The spectral features and duration-based features were derived from the forced-alignment of the manual transcription to the waveform. The classification accuracy reached $96.6 \%$ when all of the prosodic, lexical, part-of-speech, and spectral features were combined. We also computed precision $p$ (the ratio of correct assignments to a category by the system to all of the system's assignments to that category), recall $r$ (the ratio of correct assignments to a category by the system to all of the manual assignments to that category), and $F$-score $f\left(f=\frac{1}{0.5 / p+0.5 / r}\right)$ for each of the three cognitive states, and present the results in Table 8. We further present in Figure 5 the three one-class $F$-scores that were yielded using various feature sets. The figure shows that the difference in $F$-scores across the single-type feature set (i.e., prosody, lexicon, part-of-speech, or spectrum) was small for confidence classification, but turned to be larger for puzzlement classification and largest for hesitation classification. In addition, the comparison of the three one-class $F$-scores showed that when only prosody, lexicon, or part-of-speech was used, the best classification performance fell on confidence, followed by puzzlement. However, there was no distinction among the three one-class $F$ scores when spectral information was included in the feature set. 
Table 8: Precision $p$, recall $r$, and $F$-score $f$ using transcribed words. Pros $=$ Prosody, Lex $=$ Lexicon, Pos $=$ Part-of-speech, Spect $=$ Spectrum .

\begin{tabular}{|c|c|c|c|c|c|c|c|c|c|}
\hline \multirow[t]{2}{*}{ Feature } & \multicolumn{3}{|c|}{ Confidence } & \multicolumn{3}{|c|}{ Puzzlement } & \multicolumn{3}{|c|}{ Hesitation } \\
\hline & $p$ & $r$ & $f$ & $P$ & $r$ & $f$ & $p$ & $r$ & $f$ \\
\hline Pros & 0.896 & 0.942 & 0.918 & 0.791 & 0.777 & 0.784 & 0.710 & 0.484 & 0.576 \\
\hline Lex & 0.818 & 0.914 & 0.864 & 0.850 & 0.742 & 0.792 & 0.634 & 0.417 & 0.503 \\
\hline Pos & 0.712 & 0.967 & 0.820 & 0.862 & 0.479 & 0.616 & 0.357 & 0.019 & 0.036 \\
\hline Spect & 0.938 & 0.977 & 0.957 & 0.951 & 0.899 & 0.924 & 0.983 & 0.913 & 0.947 \\
\hline Pros+Spect & 0.952 & 0.988 & 0.970 & 0.971 & 0.917 & 0.944 & 0.984 & 0.909 & 0.945 \\
\hline $\begin{array}{l}\text { Pros+Lex+ } \\
\text { Pos+Spect }\end{array}$ & 0.960 & 0.987 & 0.974 & 0.971 & 0.939 & 0.955 & 0.988 & 0.919 & 0.953 \\
\hline
\end{tabular}

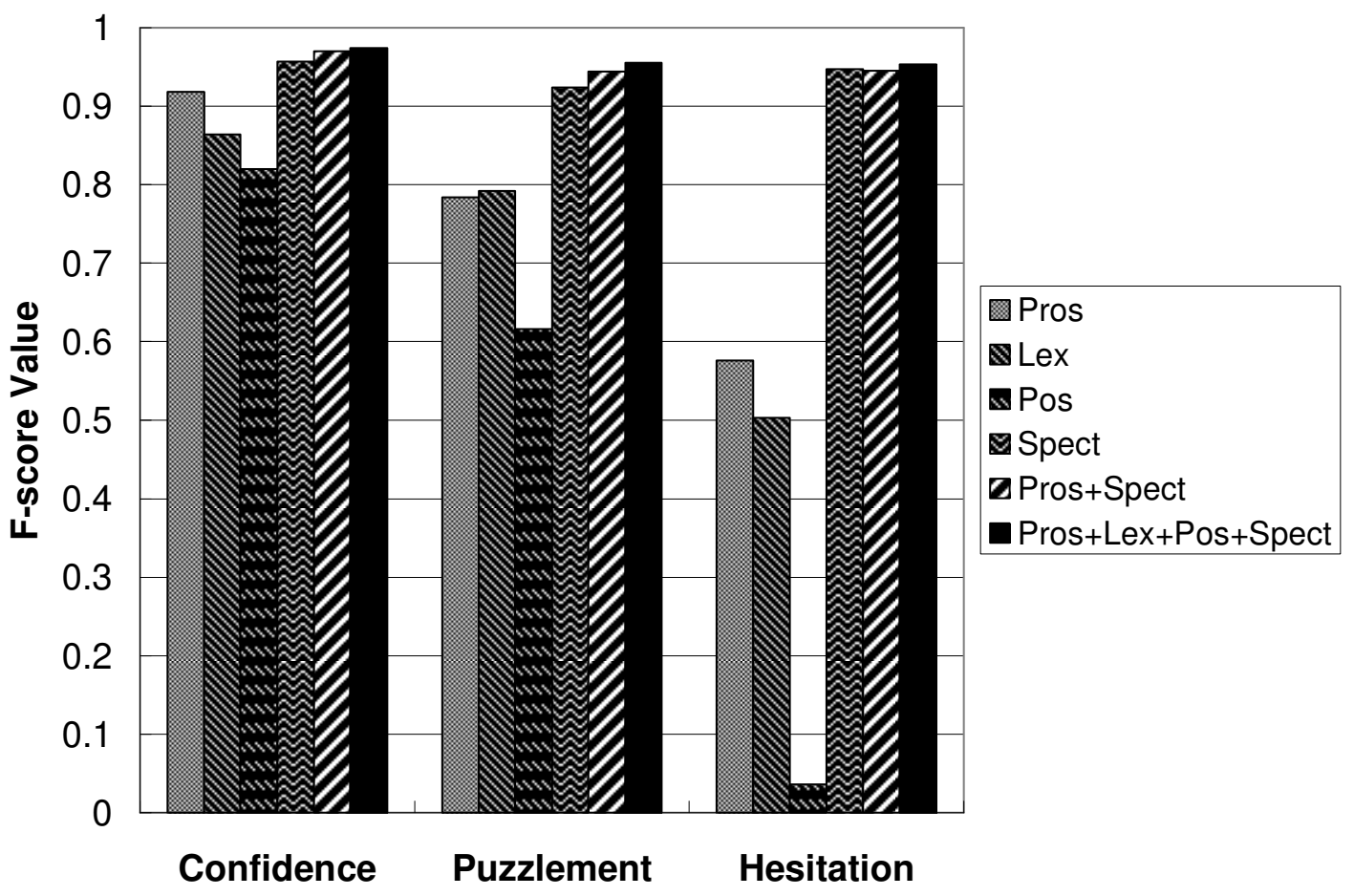

Figure 5: Classification performance with transcribed speech. Pros $=$ Prosody, Lex $=$ Lexicon, Spect $=$ Spectrum, Pos $=$ Part-of-speech . 


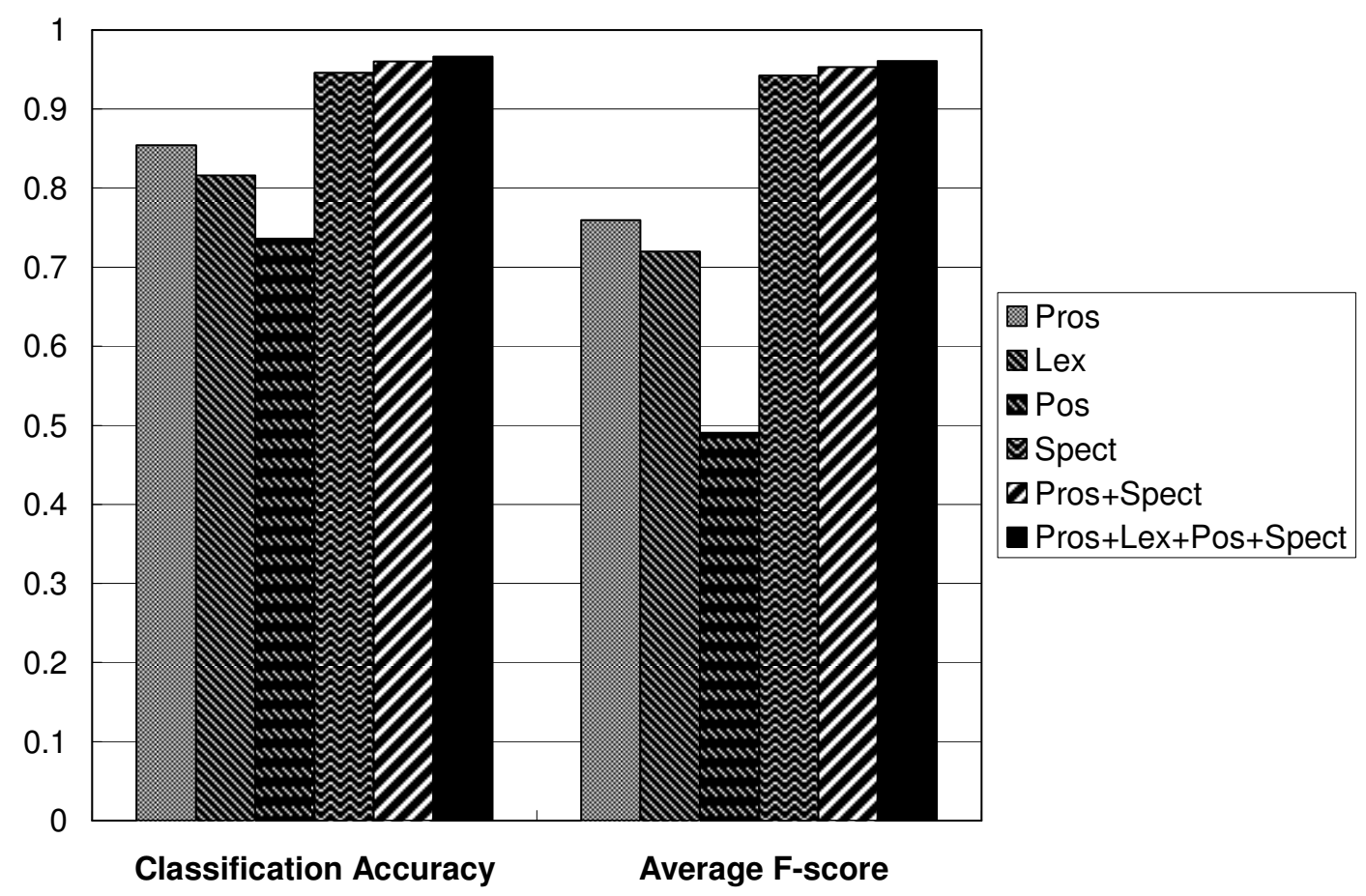

Figure 6: Feature accuracy ranking in classification of transcribed speech, in terms of classification accuracy and the average of the three one-class $F$-scores. Pros $=$ Prosody, Lex $=$ Lexicon, Pos $=$ Part-of-speech, Spect $=$ Spectrum.

In Figure 6 we evaluate the efficiency of different feature sets in terms of classification accuracy and $F$-score. Classification accuracy is the fraction of instances in the test set to be correctly classified with respect to the three classes. F-score is a harmonic mean of precision and recall: $F$-score is high only when both precision and recall are high. To globally measure classification performance across the classes, we tested the average of the $F$-scores for confidence, puzzlement and hesitation. Our results demonstrate that spectrum outperformed the other types of features in classification efficiency: the classification accuracy using spectrum outperformed prosody, lexicon, and part-of-speech by $10.8 \%, 15.9 \%$, and $28.5 \%$ relative, respectively; the average $F$-score using spectrum outperformed prosody, lexicon, and part-of-speech by $24.1 \%, 31.0 \%$, and 92.1\% relative, respectively. Compared with spectrum, the combination of all types of features only slightly increased the system performance by $2.1 \%$ relative in classification accuracy and $1.9 \%$ relative in average $F$-score. Part-of-speech played the least significant role in the classification, partly because of errors existing in the automatic part-of-speech tagger. The relatively low accuracy using text variables (lexicon and part-of-speech) 
demonstrated that the cognitive state variables were associated with speech features much more than text features. We also compared the prosodic features in terms of their occurrence frequency in the decision tree rules. Among the several features available to the classifier, the energy-based features were used most often, followed by the durationbased features.

\subsubsection{Recognized Speech}

Because the syntactic features failed to converge during machine training of recognized speech, we only evaluated the results yielded by the prosodic, lexical, and spectral features. Table 9 summarizes the classification precision, recall, and $F$-score on recognized speech, and Figure 7 shows the $F$-score of the three one-state classifications based on various feature sets. Our results show that the combination of all feature variables yielded $95.7 \%$ accuracy for recognized speech. Similar to transcribed speech, the recognition of confidence outperformed puzzlement and hesitation in terms of $F$-score when only prosodic or lexical features were used; but there was no big difference when spectral features were used. Figure 8 compares the various feature sets according to classification accuracy and average $F$-score. Our results demonstrate that spectrum played the most important role, followed by prosody, for cognitive state classification on recognized speech. Moreover, the combination of spectrum and prosody yielded almost the same results as the combination of all types of features, which demonstrated that spectrum and prosody played the decisive role in the classification. We also evaluated the prosodic features in terms of their occurrence frequency in the decision tree rules, and our results showed that duration was the most important prosodic feature, followed by energy. In contrast to many other studies (Mozziconacci and Hermes, 1998; Juang and Furui, 2000; Petrushin, 2000; Kang et al., 2000), pitch played a non-significant role in the detection of cognitive states, probably because of the frequent occurrence of pitch tracking inaccuracies in our noisy speech data. It was hard to discriminate voiced regions from unvoiced regions in the noisy recording environment. The energy of unvoiced regions carried information irrelevant to the pitch estimate, and thus the automatically extracted pitch might have contained too many pitch tracking errors to be an efficient feature. 
Table 9: Precision $p$, recall $r$, and $F$-score $f$ using recognized words. Pros $=$ Prosody, Lex $=$ Lexicon, Pos $=$ Part-of-speech, Spect $=$ Spectrum .

\begin{tabular}{|c|c|c|c|c|c|c|c|c|c|}
\hline \multirow[t]{2}{*}{ Feature } & \multicolumn{3}{|c|}{ Confidence } & \multicolumn{3}{|c|}{ Puzzlement } & \multicolumn{3}{|c|}{ Hesitation } \\
\hline & $p$ & $r$ & $f$ & $p$ & $r$ & $f$ & $p$ & $r$ & $f$ \\
\hline Pros & 0.904 & 0.940 & 0.922 & 0.811 & 0.856 & 0.833 & 0.860 & 0.474 & 0.611 \\
\hline Lex & 0.636 & 0.922 & 0.753 & 0.700 & 0.230 & 0.346 & 0.221 & 0.072 & 0.109 \\
\hline Spect & 0.889 & 0.962 & 0.924 & 0.930 & 0.834 & 0.880 & 0.876 & 0.720 & 0.790 \\
\hline Pros+Spect & 0.962 & 0.979 & 0.970 & 0.957 & 0.927 & 0.942 & 0.902 & 0.881 & 0.891 \\
\hline $\begin{array}{l}\text { Pros+Lex+ } \\
\text { Pos+Spect }\end{array}$ & 0.961 & 0.977 & 0.969 & 0.963 & 0.922 & 0.942 & 0.913 & 0.930 & 0.921 \\
\hline
\end{tabular}

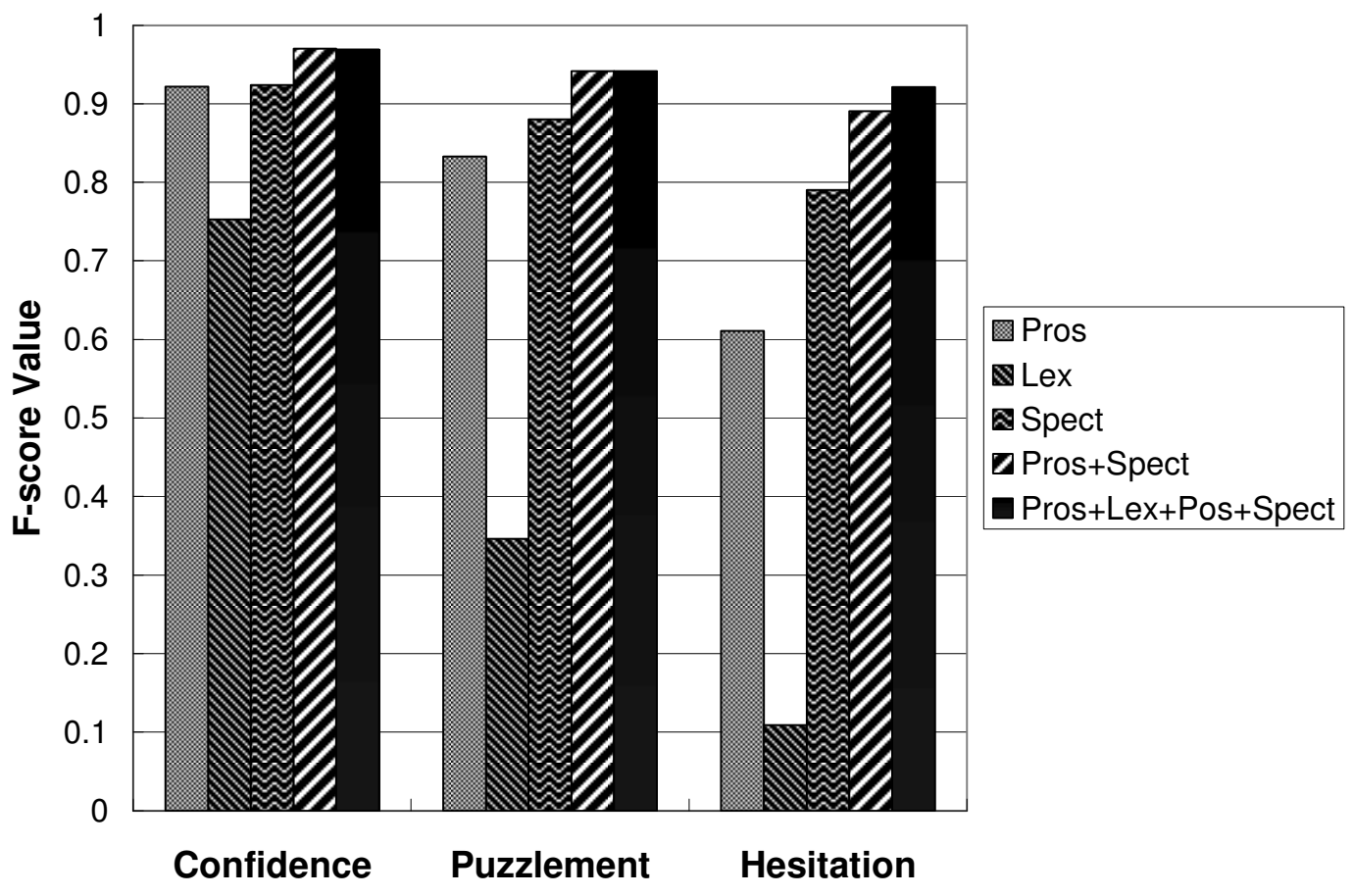

Figure 7: Classification performance with recognized speech. Pros $=$ Prosody, Lex $=$ Lexicon, Spect $=$ Spectrum, Pos $=$ Part-of-speech . 


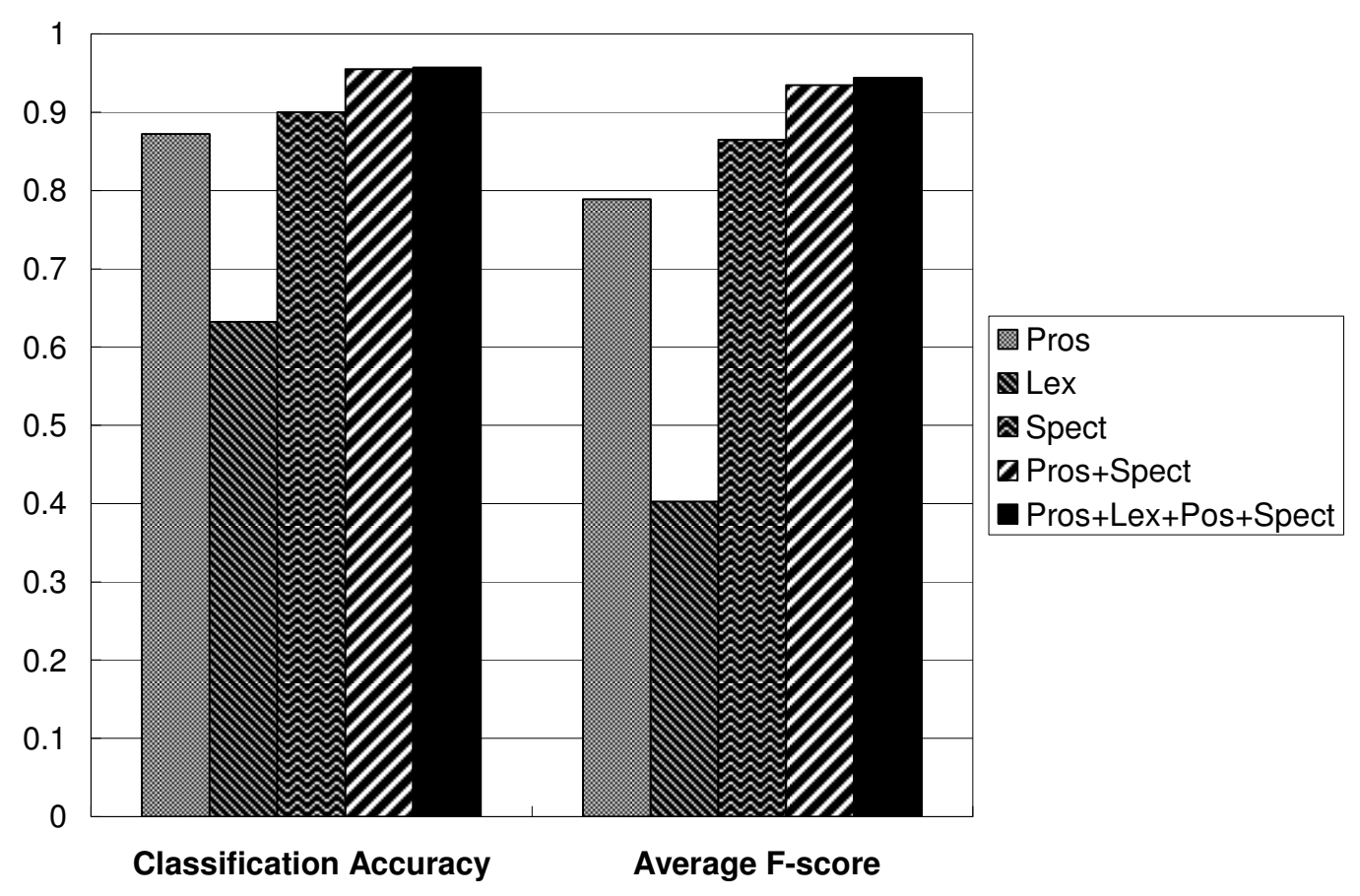

Figure 8: Feature accuracy ranking in classification of recognized speech, in terms of classification accuracy and the average of the three one-class $F$-scores. Pros $=$ Prosody, Lex $=$ Lexicon, Pos $=$ Part-of-speech, Spect $=$ Spectrum .

\subsubsection{Comparison of Transcribed Speech and Recognized Speech}

We compared the classification results using automatically recognized speech with those using manually transcribed speech to demonstrate the robustness of cognitive state classification to speech recognition errors. Table 10 lists the classification accuracy on transcribed speech, recognized speech, and the relative reduction. Our results show that spectrum and prosody were robust to speech recognition errors. The prosody-based classification accuracy was even slightly better (by $1.79 \%$ absolute) using recognized speech rather than transcribed speech, possibly because the forced-alignment procedure did not guarantee accurate estimates of word durations. Spectrum-based classification accuracy was a bit worse using recognized speech (4.65\% absolute). When spectrum and prosody were combined, cognitive state classification accuracy was almost identical for manual and automatic transcriptions. The lexicon-based classification was considerably worse using recognized speech $(18.4 \%$ absolute), demonstrating the sensitivity of keyword features to the speech recognition errors. 
Table 10: Comparison of classification correctness between transcribed speech and recognized speech. Pros $=$ Prosody, Lex $=$ Lexicon, Pos $=$ Part-of-speech, Spect $=$ Spectrum.

\begin{tabular}{cccc}
\hline Feature & $\begin{array}{c}\text { Transcribed } \\
\text { Speech }\end{array}$ & $\begin{array}{c}\text { Recognized } \\
\text { Speech }\end{array}$ & $\begin{array}{c}\text { Recognized-Transcribed } \\
\text { (\% relative) }\end{array}$ \\
\hline Pros & $85.4 \%$ & $87.2 \%$ & $2.1 \%$ \\
\hline Lex & $81.6 \%$ & $63.2 \%$ & $-22.6 \%$ \\
\hline Pos & $73.6 \%$ & - & - \\
\hline Spect & $94.6 \%$ & $90.0 \%$ & $-4.8 \%$ \\
\hline Pros+Spect & $96.0 \%$ & $95.5 \%$ & $-0.5 \%$ \\
\hline Pros+Lex+Pos+Spect & $96.6 \%$ & $95.7 \%$ & $-0.9 \%$ \\
\hline
\end{tabular}

\section{Conclusion}

This paper has proposed a three-way cognitive state classification designed to be useful for an intelligent tutorial application. We proposed classifying the cognitive activities of children users during their learning process into three categories: confidence, puzzlement and hesitation. The task was performed on 714 spontaneous utterances extracted from the audio-visual data that was collected in a Wizard-of-Oz simulation of the tutorial system. This particular cognitive state classification task could achieve an inter-annotator agreement of kappa score 0.93 , higher than emotion labelling in dialogue systems. Automatic cognitive state classification used features including keyword classes, prosody, spectrum, and syntax. Test results showed that the classification accuracy of the cognitive state classifier reached up to $96.6 \%$ for manually transcribed speech and $95.7 \%$ for automatically recognized speech, indicating that cognitive state classification is both accurate and robust to speech recognition errors. In particular, the test results showed that the proposed spectral features, measured as the difference in acoustic log likelihood between different cognitive-state dependent speech recognizers, much outperformed the other features, providing exceptionally accurate estimate of the cognitive state. Moreover, the test results showed that prosodic features and spectral features were both robust to speech recognition errors, much more than the lexical and syntactic features. In addition, among the prosodic features, the duration-based features and energy-based features were found to participate in the if-then decision more frequently than the other features; the 
pitch-based features played the least important role, possibly because of pitch tracking errors caused by the noisy recording environment.

This paper has described the task of cognitive state detection as an end in itself, but it is our goal and intention to use cognitive state detection as part of a larger automatic speech understanding system for the intelligent tutorial application. In considering possible applications of this work, we propose that an automatic labelling scheme that can be computed with an accuracy of $95.7 \%$ would probably be a useful input feature for further stages of automatic speech understanding and dialogue control.

\section{Acknowledgement}

We would like to thank Brian Pianfetti for providing us with the ITS Wizard-of-Oz audio data, and Thomas S. Huang for providing us with all kinds of help in our work. This work is supported by NSF grant number 0085980. Statements in this paper reflect the opinions and conclusions of the authors, and are not endorsed by the NSF.

\section{REFERENCES}

Alpert, S. R., Singley, M. K., and Carroll, J. M. 1999. Multiple instructional agents in an intelligent tutoring system. Intl. Workshop on Instructional Uses of Animated and Personified Agents (at the $9^{\text {th }}$ Intl. Conf. on AI in Education). Le Mans, France.

Ang, J., Dhillon, R., Krupski, A., Shriberg, E., Stolcke, A. 2002. Prosody-based automatic detection of annoyance and frustration in human-computer dialog. Proc. of Intl. Conf. on Spoken Language Processing. Denver, CO.

Batliner, A., Fischer, K., Huber, R., Spilker, J., and Nöth, E. 2003. How to find trouble in communication. Speech Communication, 40: 117-143.

Batliner, A., Huber, R., Niemann, H., Nöth, E., Spilker, J., and Fischer, K. 2000. The recognition of emotion. In W. Wahlster (ed.) Verbmobil: Foundations of Speech-toSpeech Translations. Springer, New York, Berlin.

Beck, J., Jia, P., and Mostow, J. 2003. Assessing student proficiency in a reading tutor that Listens. Proc. of the $9^{\text {th }}$ Intl. Conf. on User Modelling. Johnstown, PA.

Clark, B., Fry, J., Ginzton, M., Peters, S., Pon-Barry, H., Thomsen-Gray, Z. 2001. A multimodal intelligent tutoring system for shipboard damage control. Proc. of 2001 Intl. Workshop on Information Presentation and Multimodal Dialogue. Verona, Italy.

Cole, R., van Vuuren, S., Pellom, B., Hacioglu, K., Ma, J., Movellan, J., Schwartz, S., Wade-Stein, D., Ward, W., Yan, J. 2003. Perceptive animated interfaces: first steps toward a new paradigm for human-computer interaction. Proc. of IEEE: Special Issue on Multimodal Human Computer Interface.

Corbett, A. T. and Anderson, J. R. 1992. The Lisp intelligent tutoring system: research in skill acquisition. In J. Larkin and R. Chabay (eds.) Computer Assisted Instruction and Intelligent Tutoring System: Shared Goals and Complementary Approaches. Lawrence Eflbaum, Hillsdale, NJ. 
Crowley, R., Medvedeva, O., and Jukic, D. 2003. SlideTutor: a model-tracing intelligent tutoring system for teaching microscopic diagnosis. Proc. of the $11^{\text {th }}$ Intl. Conf. on Artificial Intelligence in Education. Sydney, Australia.

Fernandez, R. and Picard, R. W. 2003. Modeling driver's speech under stress. Speech Communication, 40: 145-159.

Flammia, G. 1998. Discourse segmentation of spoken dialogue: an empirical approach. Ph.D. Thesis. MIT.

Forbes-Riley, K. and Litman, D. 2004. Predicting emotion in spoken dialogue from multiple knowledge sources. Proc. of Human Language Technology Conference/North American Chapter of the Association for Computational Linguistics Annual Meeting. Boston, MA.

Gobl, C. and Chasaide, A. N. 2003. The role of voice quality in communicating emotion, mood and attitude. Speech Communication, 40: 189-212.

Godfrey, J.J., Holliman, E.C., and McDaniel, J., 1991. SWITCHBOARD: Telephone speech corpus for research and development. Proc. of IEEE Intl. Conf. on Acoustics, Speech, and Signal Processing. Toronto, Canada.

Graesser, A. C., Lehn, K. V., Rose, C. P., Jordan, P. W., and Harter, D. 2001. Intelligent tutoring system with conversational dialogue. AI Magazine, 22(4): 39-52.

Juang, B.-H. and Furui, S. 2000. Automatic recognition and understanding of spoken language - a first step towards natural human-machine communication. Proc. of IEEE, 88(8): 1142-1165.

Jurafsky, D., Bates, R., Coccaro, N., Martin, R., Meteer, M., Ries, K., Shriberg, E., Stolcke, A., Taylor, P., and Ess-Dykema, C. V. 1997. Switchboard discourse language modeling project final report. Johns Hopkins LVCSR Workshop. Baltimore, MD.

Kafai, Y. and M. Resnick. 1996. Constructionism in Practice: Designing, Thinking and Learning in a Digital World. Lawrence Erlbaum Associates, Mahwah, NJ.

Kang, B.-S., Han, C.-H., Lee, S.-T., Youn, D.-H., Lee, C. 2000. Speaker dependent emotion recognition using speech signals. Proc. of Intl. Conf. on Spoken Language Processing. Beijing, China.

Katz, S. M. 1987. Estimation of probabilities from sparse data for the language model component of a speech recognizer. IEEE Trans. Acoustics, Speech and Signal Processing, 35(3): 400-401.

Kitazawa, S., Ichikawa, H., Kobayashi, S., and Nishinuma, Y. 1997. Extraction and representation rhythmic components of spontaneous speech. Proc. of European Conf. on Speech Communication and Technology. Rhodes, Greece.

Lee, C. M. and Narayanan, S. 2005. Toward detecting emotions in spoken dialogs. IEEE Trans. on Speech and Audio Processing, 13(2): 293-303.

Lee, L. and Rose, R. 1998. A frequency warping approach to speaker normalization. IEEE Trans. on Speech and Audio Processing, 6(1): 49-59.

Lee, S., Potamianos, A., and Narayanan, S. 1999. Acoustics of children's speech: developmental changes of temporal and spectral parameters. The Journal of the Acoustical Society of America, 105(3): 1455-1468.

Lesgold, A., Lajoie, S., Bunzo, M., and Eggan, G. 1990. Sherlock: a coached practice environment for an electronics troubleshooting job. In J. Larkin, R. Chabay, and C. Sheftic (eds.) Computer Assisted Instruction and Intelligent Tutoring Systems Establishing Communication and Collaboration. Erlbaum, Hillsdale, NJ. 
Li, Q., Zheng, J., Tsai, A., and Zhou, Q. 2002. Robust endpoint detection and energy normalization for real-time speech and speaker recognition. IEEE Trans. on Speech and Audio Processing, 10(3): 146-157.

Lieberman, P. and Michaels, S. B. 1962. Some aspects of fundamental frequency and envelope amplitude as related to the emotional content of speech. Journal of the Acoustical Society of America, 34: 922-927.

Litman, D., Rose, C., Forbes-Riley, K., VanLehn, K., Bhembe, D., and Silliman, S. 2004. Spoken versus typed human and computer dialogue tutoring. Proc. of the $7^{\text {th }}$ Intl. Conf. on Intelligent Tutoring Systems. Maceió, Brazil.

Litman, D. and Silliman, S. 2004. ITSPOKE: an intelligent tutoring spoken dialogue system. Proc. of the Human Language Technology Conference/North American Chapter of the Association for Computational Linguistics Annual Meeting. Boston, MA.

Martinovsky, B. and Traum, D. 2003. Breakdown in human-machine interaction: the error is the cue. Proc. of the ISCA Tutorial and Research Workshop on Error Handling in Dialogue Systems. Château-d'Oex, Switzerland.

Morgan, N. and Fosler-Lussier, E. 1998. Combining multiple estimators of speaking rate. Proc. of IEEE Intl. Conf. on Acoustics, Speech, and Signal Processing. Seattle, WA.

Mostow, J., Beck, J., Winter, S. V., Wang, S., and Tobin, B. 2002. Predicting oral reading miscues. Proc. of Intl. Conf. on Spoken Language Processing. Denver, CO.

Mozziconacci, S. and Hermes, D. 1998. Study of intonation patterns in speech expressing emotion or attitude: production and perception. IPO Annual Progress Report. IPO, Eindhoven.

Munoz, M., Punyakanok, V. Roth, D., and Zimak, D. 1999. A learning approach to shallow parsing. Proc. of Conf. on Empirical Methods in Natural Language Processing and Very Large Corpora. College Park, MD.

Narayanan, S., and Potamianos, A. 2002. Creating conversational interfaces for children. IEEE Transactions on Speech and Audio Processing, 10(2): 65-78.

Pellom, B., Ward, W., and Pradhan, S. 2000. The CU communicator: an architecture for dialogue systems. Proc. of Intl. Conf. on Spoken Language Processing. Beijing, China.

Petrushin, V. 1999. Emotion in speech: recognition and application to call centers. In C. Dagli, A. Buczak, J. Ghosh, M. J. Embrechts, and O. Ersoy (eds.) Intelligent Engineering Systems Through Artificial Neural Networks, 9: 1085-1092.

Petrushin, V. A. 2000. Emotion recognition in speech signal: experimental study, development, and application. Proc. of Intl. Conf. on Spoken Language Processing. Beijing, China.

Polzin, T. S. and Waibel, A. 2000. Emotion-sensitive human-computer interfaces. ISCA Workshop on Speech and Emotion: a Computational Framework for Research. Newcastle, Northern Ireland.

Pon-Berry, H., Clark, B., Bratt, E. O., Schultz, K. and Peters, S. 2004. Evaluating the effectiveness of SCoT: a spoken conversational tutor. Proc. of ITS 2004 Workshop on Dialogue-based Intelligent Tutoring Systems. Maceio, Brazil.

Potamianos, A. and Narayanan, S. 2003. Robust recognition of children's speech. IEEE Trans. Speech and Audio Processing, 11: 603-616.

Reyes, R. L., Galvey, C., Gocolay, M. C., Ordona, E., and Ruiz, C. 2000. Multimedia intelligent tutoring system for context-free grammar. Proc. of Philippine Computing Science Congress. Manila, Philippines. 
Rulequest Research. 2004. Data Mining Tools. http://www.rulequest.com/see5-info.html

Schultz, K., Bratt, E. O., Clark, B., Peters, S., Pon-Barry, H., Treeratpituk, P. 2003. A scalable, reusable spoken conversational tutor: SCoT. AIED 2003 Supplementary Proceedings. Sydney, Australia.

Steele, M. M. and Steele, J. W. 1999. Discover: an intelligent tutoring sytem for teaching students with learning difficulties for solve word problems. Journal of Computers in Mathematics and Science Teaching, 18(4): 351-359.

Ward, W. and Pellom, B. 1999. The CU communicator system. IEEE Workshop on Automatic Speech Recognition and Understanding. Keystone, CO.

Wilensky, U. 1991. Abstract meditations on the concrete. In I. Harel and S. Papert (eds.) Constructionism. Ablex, Norwood, NJ.

Young, S., Kershaw, D., Odell, J., Ollason, D., Valtchev, V., Woodland, P. 2000. The HTK Book. http://htk.eng.cam.ac.uk/

Zhan, P. and Westphal, M. 1997. Speaker normalization based on frequency warping. Proc. of IEEE Intl. Conf. on Acoustics, Speech, and Signal Processing. Munich, Germany.

Zue, V., Seneff, S., Glass, J., Polifroni, J., Pao, C., Hazen, T., and Hetherington, L. 2000. Jupiter: a telephone-based conversational interface for weather information. IEEE Trans. on Speech Audio Processing, 8: 85-96. 